result may be limited by small sample size. In type I IFNGS test-high pts, a log-linear logistic model was used to describe the treatment effect of anifrolumab. Pt dropouts were more likely in nonresponders. Clinical simulations demonstrated dosages $<300 \mathrm{mg}$ would result in inadequate PK exposure and suboptimal efficacy in some pts with SLE. In contrast, simulations indicated minimal efficacy improvement for dosages $>300 \mathrm{mg}$, consistent with the Phase Ilb MUSE study outcomes.

Conclusions: Based on $\mathrm{E}-\mathrm{R}$ analyses and overall risk assessment, a 300 $\mathrm{mg}$ Q4W, intravenous dosage regimen is recommended for pivotal anifrolumab studies in pts with SLE.

References:

[1] Furie R, et al. Arthritis Rheumatol. 2017;69:376-86.

[2] Wang B, et al. Clin Pharmacol Ther. 2013;93:483-92.

Acknowledgements: Funded by Medlmmune. Medical writing support: R. Plant, QXV Comms, an Ashfield company, funded by Medlmmune.

Disclosure of Interest: L. Santiago Shareholder of: AstraZeneca, Employee of: Medlmmune LLC, B. Wang Shareholder of: AstraZeneca, Employee of: Medlmmune LLC, P. Brohawn Shareholder of: AstraZeneca, Employee of: Medlmmune LLC, L. Wang Employee of: Medlmmune LLC, G. Illei Shareholder of: AstraZeneca, Employee of: Medlmmune LLC, L. Roskos Shareholder of: AstraZeneca, Employee of: AstraZeneca

DOI: 10.1136/annrheumdis-2017-eular.3517

\section{SAT0244 A LONG-TERM FOLLOW-UP STUDY OF ALLOGENEIC MESENCHYMAL STEM CELLS TRANSPLANTATION IN PATIENTS WITH DRUG-RESISTANT SYSTEMIC LUPUS ERYTHEMATOSUS}

D. Wang ${ }^{1}$, L. Sun ${ }^{2} .{ }^{1}$ Department of Rheumatology and Immunology; ${ }^{2}$ Department of Rheumatology, The Affiliated Drum Tower Hospital of Nanjing University Medical School, Nanjing, China

Background: Allogeneic mesenchymal stem cells (MSC) transplantation showed therapeutic effect in active and refractory systemic lupus erythematosus (SLE). Objectives: To determine the long-term safety and efficacy of allogeneic MSC transplantation (allo-MSCT) in severe SLE patients refractory to previous therapies.

Methods: All consecutive SLE patients who received at least one allo-MSCT were analyzed. SLEDAI score of more than or equal to 8 or with at least one BILAG grade A or at least two BILAG grade B manifestations. All the patients were refractory to corticosteroid and/or immunosuppressive drugs treatment. Allogeneic bone marrow and/or umbilical cord derived MSCs were infused intravenously, with one million cells per kilogram of bodyweight for each infusion. The primary end point was 5 -year overall survival. Secondary end points included complete clinical remission, partial clinical remission and relapse.

Results: Eighty-one patients were enrolled and underwent allo-MSCT. Thirteen patients died within 5 years post-MSCT and the 5 -year overall survival rate was $84 \%(68 / 81)$. At 5 -year follow-up, 22 patients $(22 / 68,32 \%)$ were in complete clinical remission and another 6 patients $(6 / 68,9 \%)$ were in partial clinical remission, and the 5 -year disease remission was $41 \%$ (28/68). In total, 37 patients had achieved clinical remission (27 in complete remission and 10 in partial remission) at the 5 years visit and then 9 patients subsequently relapsed. The 5-year overall rate of relapse was $24 \%(9 / 37)$. SLEDAl scores, levels of serum albumin and complement $\mathrm{C} 3$, peripheral white blood cell and platelet numbers, as well as proteinuria levels continued to improve during the follow-up.

Conclusions: Allogeneic MSCT is safe and resulted in long-term clinical remission in severe and drug-resistant SLE patients.

Disclosure of Interest: None declared

DOI: 10.1136/annrheumdis-2017-eular.2656

\section{SAT0245 SERUM IFN GAMA MAY PREDICT THERAPEUTIC EFFECT OF MESENCHYMAL STEM CELLS TRANSPLANTATION IN SYSTEMIC LUPUS ERYTHEMATOSUS PATIENTS}

D. Wang, L. Sun. Department of Rheumatology, The Affiliated Drum Tower Hospital of Nanjing University Medical School, Nanjing, China

Background: Umbilical cord (UC) derived mesenchymal stem cells (MSCs) show immunoregulatory properties on various immune cells and display clinical effect on lots of autoimmune disease like systemic lupus erythematosus (SLE).

Objectives: The aim of this study is to investigate the effect of SLE environment on UC MSCs and to observe the possible serum biomarker to predict the therapeutic effect.

Methods: UC MSCs were co-cultured with peripheral blood mononuclear cells (PBMC) from active lupus patients at a ratio of 1:4. The proliferation, apoptosis and surface markers of UC MSCs were observed. UC MSCs functional molecules were assessed by real-time PCR and the signaling pathways were analyzed by western blot. Different recombinant cytokines were used to stimulate UC MSCs in vitro and the functional factors were determined by real-time PCR. In the last, twenty-six patients with SLE, refractory to conventional therapies, were given UC MSCs transplantation. The clinical effect was followed-up for one year to classify responder and non-responder groups, and baseline serum cytokines were analyzed by ELISA.
Results: The co-culture of lupus patients PBMC had no effect on UC MSCs surface markers and apoptosis, but promoted MSCs proliferation. Lupus PBMC were more prone to stimulate UC MSCs to secret VEGF as well as CXCL-12, compared to PBMC from normal controls. Furthermore, lupus PBMC activated Akt, IKB and Stat5 signaling pathways in UC MSCs but not affect Erk1/2 and Smad1/5/8 pathways. When stimulated by different cytokines, we found that interferon $\gamma(\mathrm{IFN}-\gamma)$ was still the most important cytokine to induce IDO1 as well as IDO2 productions in UC MSCs; both had dose-dependent manners. Moreover, our clinical study showed that baseline higher levels IFN- $\gamma$ might predict a good response to MSCs transplantation in active lupus patients.

Conclusions: Baseline IFN- $\gamma$ levels may predict clinical response to MSCs therapy for active lupus patients, which will help us to choose appropriate patient for clinical transplantation.

Disclosure of Interest: None declared

DOI: 10.1136/annrheumdis-2017-eular.2661

\section{SAT0246 EXPLORER STUDY: RITUXIMAB USE IN SYSTEMIC LUPUS ERYTHEMATOSUS, A NEW LOOK ON OLD DATA}

M. Scherlinger, C. Carcaud, M.E. Truchetet, P. Dufau, L. Couzy, J. Seneschal, T. Barnetche, E. Lazaro, C. Richez. Fhu Acronym, CHU de Bordeaux, Bordeaux, France

Background: Even if randomized trials EXPLORER and LUNAR failed to prove the superiority of rituximab versus placebo in patients with systemic lupus erythematosus, several encouraging indications as refractory lupus nephritis and new clinical trials renewed interest for this molecule.

We hypothetized that SLE response criteria used in EXPLORER were not sensible enough to show rituximab efficacy and that new response criteria could show a significant difference.

Objectives: Our objective was to reanalyze EXPLORER trial's raw data using the newly described SLE response criteria.

Methods: We proceeded to a pre-specified re-analyze of EXPLORER trial's raw data. The patients included in EXPLORER study had active SLE disease defined by a British Isles Lupus Assessment Group (BILAG) score A or 2 BILAG B despite immunosuppressive regimen. Renal and neurological SLE were excluded. Patients were randomized through a 2/1 ratio to receive either two 1 gr rituximab infusions (day 0 and 15) repeated at month 6 or a placebo. Standard SLE treatment and other immunosuppressant were continued. Patients received in a stratified manner prednisone ranging from 0.5 to $1.0 \mathrm{mg} / \mathrm{kg}$ depending on disease severity at inclusion. The original efficacy criterion was a composite clinical score using BILAG at week 52.

In our new analysis, rituximab efficacy was assessed at week 52 using 4 criteria: SRI-4 (Systemic lupus erythematosus Responder Index) with and without a concomitant oral prednisone (OP) tapering objective of $<10 \mathrm{mg}$ at month 6 (SRI-4 with and without OP tapering), Lupus Low Disease Activity Score (LLDAS) and BILAG-based Combined Lupus Assessment (BICLA).

Results: Data from all 257 patients were available. There was 234 women $(91 \%)$ with a mean age of 40,3 years among which $177(69 \%)$ received hydroxychloroquine.

At week 52 , SRI-4 response rate was $27,2 \%$ in the rituximab group vs $22,7 \%$ in the placebo group $(\mathrm{p}=0.43)$; SRI-4 with OP tapering was $16 \%$ in the rituximab group vs $13.6 \%$ in the placebo group ( $p=0.62)$; LLDAS was $16 \%$ in the rituximab group vs $12.5 \%$ in the placebo group $(p=0.46)$ and BICLA was $15.4 \%$ in the rituximab group vs $15.9 \%$ in the placebo group $(p=0.91)$.

Subgroup analyses demonstrated a trend for better efficacy of rituximab compared to placebo in the subgroup of patients co-treated with methotrexate: SRI-4 of $30.6 \%$ in the rituximab group vs $12 \%$ in the placebo group $(n=74, p=0.08)$. This trend was not found in the subgroup co-treated with azathioprine: SRI-4 of $26.7 \%$ in the rituximab group vs $30.6 \%$ in the placebo group $(p=0.68)$, nor in the subgroup co-treated with mycophenolic acid: SRI-4 of $23.1 \%$ in the rituximab group vs $21.6 \%$ in the placebo group $(p=0.86)$. In the subgroup of patients with a BILAG $\mathrm{A} / \mathrm{B}$ in hematological system or vasculitis at baseline, there was a significantly higher SRI-4 response rate with rituximab: $28,6 \%$ vs $5,3 \%$ in the hematological group $(n=61, p=0,047)$ and $39,3 \%$ vs $0 \%$ in the vasculitis group $(n=38, p=0,037)$. Cumulative dose of steroids at week 52 were not statistically different: $4223 \mathrm{mg}$ in the rituximab group vs $4390 \mathrm{mg}$ in the placebo group $(p=0,65)$.

Conclusions: Our study confirms the results from the original EXPLORER study. These results might be partly related to the study's design, notably the high daily oral prednisone used. Our work suggests efficacy in subgroups with vasculitis and hematological involvement.

Disclosure of Interest: None declared

DOI: 10.1136/annrheumdis-2017-eular.1785 